\title{
Age, Gender and Geographic Differences in Global Health Burden of Cirrhosis and Liver Cancer due to Nonalcoholic Steatohepatitis
}

\author{
Tianyue Zhang ${ }^{1^{*}}$, Jingya $\mathrm{Xu}^{1^{*}}$, Lingxia Ye ${ }^{1^{*}}$, Xiling Lin ${ }^{1}$, Yufeng $\mathrm{Xu}^{2}$, Xiaowen Pan ${ }^{1}$, Xifang Weng ${ }^{3}$, Chuyu Ye ${ }^{3}$, \\ Longjiang Fan $^{3}$, Yuezhong Ren ${ }^{\circledR}$ and Peng-Fei Shan ${ }^{1 凶}$ \\ 1. Department of Endocrinology, the Second Affiliated Hospital of Zhejiang University School of Medicine, 88 Jiefang Road, Hangzhou, Zhejiang, 310009, China. \\ 2. Department of Ophthalmology, the Second Affiliated Hospital of Zhejiang University School of Medicine, 88 Jiefang Road, Hangzhou, Zhejiang, 310009, China. \\ 3. Institute of Crop Sciences and Institute of Bioinformatics, Zhejiang University, Hangzhou, Zhejiang, 310058, China. \\ *These authors contributed equally to this work.
}

Corresponding authors: Peng-Fei Shan, Department of Endocrinology, the Second Affiliated Hospital of Zhejiang University School of Medicine, 88 Jiefang Road, Hangzhou, Zhejiang, 310009, China; Phone: +86-0571-87784623; E-mail: pengfeishan@zju.edu.cn. Yuezhong Ren, Department of Endocrinology, the Second Affiliated Hospital of Zhejiang University School of Medicine, 88 Jiefang Road, Hangzhou, Zhejiang, 310009, China; Phone: +86-0571-87783551; E-mail: renyuez@zju.edu.cn.

() The author(s). This is an open access article distributed under the terms of the Creative Commons Attribution License (https://creativecommons.org/licenses/by/4.0/). See http://ivyspring.com/terms for full terms and conditions.

Received: 2020.08.22; Accepted: 2021.02.18; Published: 2021.03.15

\begin{abstract}
Objective: Recently, Nonalcoholic Steatohepatitis (NASH) has become a major contributor to cirrhosis and liver cancer. Therefore, the Global Burden of Disease (GBD) 2017 was used to comprehensively analyze the global, regional, and national burden of cirrhosis and liver cancer due to NASH between 1990 and 2017.

Methods: Data for cirrhosis and liver cancer due to NASH were extracted from the GBD study 2017. Socio-demographic Index (SDI) in 2017 was cited as indicators of socioeconomic status. ARIMA model was established to forecast the future health burden. Kruskal-Wallis test and Pearson linear correlation were adopted to evaluate the gender disparity and association with socioeconomic level.

Results: From 1990-2017, the global disability-adjusted life years (DALYs) numbers of liver cancer due to NASH increased from 0.71 million to 1.46 million. The age-standardized DALYs rates of liver cancer due to NASH were negatively associated with SDI levels $(r=0 .-409, p<0.001)$. Geographically, Australasia experienced the largest increase in the burden of liver cancer due to NASH, with the age-standardized DALYs rate increasing by $143.54 \%$. The global prevalence number of liver cancer due to NASH peaked at 60-64 years in males and at 65-69 years in females. Globally, the burden was heavier in males compared with females. Male-female-ratio of age-standardized DALYs rates in liver cancer due to NASH were positively related to SDI $(r=0.303, P=0.011)$
\end{abstract}

Conclusion: The global burden of NASH-associated liver cancer has increased significantly since 1990, with age, gender and geographic disparity. Public awareness of liver diseases due to NASH should be emphasized.

Key words: nonalcoholic steatohepatitis; cirrhosis; liver cancer; disability-adjusted life years; health burden; Sociodemographic Index

\section{Introduction}

Nonalcoholic fatty liver disease (NAFLD) is a group of acquired metabolic-related liver diseases characterized by the presence of hepatic fat accumulation and the exclusion of other causes of hepatic steatosis [1]. With the rising prevalence of obesity, NAFLD has become a heavy burden for personal and public health [1, 2]. Nonalcoholic steatohepatitis (NASH) occurs in approximately 59\% of patients with NAFLD, which is diagnosed by liver biopsy findings of chronic liver injury and inflammation plus steatosis [3]. NASH is rapidly becoming a major contributor to cirrhosis and liver cancer [4-6]. In an 8-year period, $21-26 \%$ of NASH progressed to cirrhosis and $13 \%$ progressed directly to liver cancer [7-9].

To our knowledge, few studies on the burden of cirrhosis and liver cancer due to NASH by time, age, gender, region or socioeconomic status have been 
published. The health burden of cirrhosis and liver cancer due to NASH has been quantified by disability-adjusted life years (DALYs) in the Global Burden of Disease (GBD) study [10]. DALYs, defined as the sum of years lived with disability and years of life lost owing to premature death, combined both the prevalence of a disease and its impact on mortality and morbidity [10]. Thus, it could illustrate the whole picture of a disease and provide hints for public health policy development. Accordingly, in the present study, we sought to investigate the changes in global health burden of cirrhosis and liver cancer due to NASH from 1990 to 2017, and the age, gender and geographic differences in the health burden, based on the GBD study 2017.

\section{Materials and Methods}

\section{Data source}

The GBD study 2017 provides a detailed epidemiologic assessment (including incidence, prevalence, mortality and DALYs etc.) of 345 diseases and injuries and 84 risk factors by age and sex on a global scale and for 195 countries and territories spanning the years from 1990 to 2017. The detailed methods about inputs, analytical processes, and outputs have been described in previous paper elsewhere [11-16]. Briefly, the study used a total of 68,781 data sources (including published literature, surveillance data, survey data, hospital and clinical data, and other types of data), maximized the comparability of data and provided a standard approach for estimating disease burden. Citations for all data sources in GBD 2017 are provided in searchable form through a web tool (https://gbd2017. healthdata.org/gbd-search/). An integrative Bayesian meta-regression method that estimates a generalized negative binomial model for all epidemiological data was used through DisMod-MR 2.1 in the computation of GBD estimates of disease burden.

Data for cirrhosis and liver cancer due to NASH were extracted from the GBD study 2017. In GBD study 2017, cirrhosis and liver cancer due to NASH were defined only if NASH was listed as a specific etiology for cirrhosis and liver cancer. All GBD data used in this study were collected from the Global Health Data Exchange [10] which comprised: (1) Global total and gender-specific data on incidence, prevalence, death and DALYs, as absolute numbers, and age-standardized rates (per 100000 population), annually from 1990 to 2017; (2) Global gender-age-specific prevalence and DALYs data, as absolute numbers in 2017; (3) GBD regions' total and gender-specific DALYs data in 1990 and 2017, as age-standardized rates; (4) National total and gender-specific DALYs data, as absolute numbers and age-standardized rates in 2017; (5) Gender-specific age-standardized DALYs rates by Socio-demographic Index (SDI) levels in 2017. Ethical approval and informed consent were not required for this study because of public accessibility of the data.

\section{Socioeconomic status}

The SDI was calculated by the GBD study 2017 as a composite indicator of development status strongly correlated with health outcomes [10]. In short, it is the geometric mean of 0 to 1 indices of total fertility rate under the age of 25 (TFU25), mean education for those aged 15 and older (EDU15+), and lag distributed income (LDI) per capita, with a higher value implying a higher level of socioeconomic development. Countries or territories were divided into five groups based on their SDI values in 2017 (See the Supplementary Table S3): High SDI (> 0.81), high-middle SDI (0.70-0.81), middle SDI (0.61-0.70), low-middle SDI (0.46-0.60), and low SDI $(<0.46)$.

\section{Forecasting cirrhosis and liver cancer due to NASH burden beyond 2017}

Auto-Regressive Integrated Moving Average (ARIMA) model was widely used in epidemiological study for forecasting future trend [17]. ARIMA model was established in $\mathrm{R}$ language to forecast cirrhosis and liver cancer due to NASH burden from 2018 towards 2050 (See the Supplementary Statistical Analysis Methods for details).

\section{Statistical analysis}

Data are expressed as a value with a 95\% uncertainty interval (UI). Age-standardized rates are expressed as the number per 100000 population. The Kruskal-Wallis test was adopted to assess differences in global age-standardized prevalence and DALYs rates for each SDI-based country group. Association of gender difference (male-female ratio) in agestandardized DALYs rates with SDI were tested via Pearson correlation and Linear regression analyses. All statistical analyses, except as otherwise specified, were conducted with Prism software (version 8; GraphPad). A P-value less than 0.05 was considered statistically significant.

\section{Results}

\section{Temporal trends of burden of cirrhosis and liver cancer due to NASH from 1990 to 2017}

As shown in Figure 1 and Figure S1, from 1990-2017, increasing trends of global burden of cirrhosis and liver cancer due to NASH were observed in incidence number, prevalence number, death number, DALYs number, age-standardized 
incidence rate and age-standardized prevalence rate. For the global age-standardized death rate and DALYs rate of cirrhosis due to NASH, we observed a decrease from 2005 to 2013 and an increase from 2013 to 2017. Meanwhile, for the global age-standardized death rate and DALYs rate of liver cancer due to NASH, we observed a decrease from 2003 for death rate and 2000 for DALYs rate to 2007, a plateau from 2007 to 2013, and an increase from 2013 to 2017.

Overall, as shown in Table 1, the global all-age incidence of cirrhosis due to NASH was 367,779 $(334,460-403,729)$ cases with an age-standardized rate of $4.6(4.2-5.1)$ per 100,000 in 2017. The cirrhosis due to NASH prevalence cases were 892.32 million $(95 \%$ UI: 858.62-927.95 million) and the age-standardized rate was $11,060.5$ (95\% UI: $10651.3-11493.1$ ) per 100,000 in 2017. The cirrhosis due to NASH death number was $118,030(108,618-128,577)$ with an age-standardized rate of 1.5 (1.3-1.6) per 100,000 in 2017. As for DALYs, the all-age DALYs numbers were 3.43 million $(95 \%$ UI: 3.15-3.74 million) with an age-standardized rate of 41.8 (38.5-45.5) per 100,000 in 2017.

The global all-age incidence of liver cancer due to NASH was $72,197(64,602-79,915)$ cases with an age-standardized rate of $0.9(0.8-1.0)$ per 100,000 in
2017. The liver cancer due to NASH prevalence cases were 97,376 (95\% UI:86,789-108,049) and the agestandardized rate was 1.2 (1.1-1.3) per 100,000 in 2017. The liver cancer due to NASH death number was $66,875(59,560-74,511)$ with an age-standardized rate of $0.8(0.8-0.9)$ per 100,000 in 2017. As for DALYs, the all-age DALYs numbers were 1.46 million (95\% UI: 1.30-1.62 million) with an age-standardized rate of 18.0 (16.1-20.0) per 100,000 in 2017.

\section{Age and gender differences in burden of cirrhosis and liver cancer due to NASH}

The age and sex-specific prevalence and DALYs number for cirrhosis and liver cancer due to NASH were shown in Figure 2. For both males and females, age group (45-49 years) had the highest prevalence numbers of cirrhosis due to NASH. For males, age group (60-64 years) had the highest prevalence numbers of liver cancer due to NASH. For females, age group (65-69 years) had the highest prevalence numbers of liver cancer due to NASH. The prevalence and DALYs number were higher in males than females. At older age groups above 70 years, the DALYs number in females for cirrhosis due to NASH started to be higher than males.

Table 1. Global burden of cirrhosis and liver cancer due to NASH in 1990 and in 2017

\begin{tabular}{|c|c|c|c|c|c|c|}
\hline & \multicolumn{3}{|l|}{ Cirrhosis due to NASH } & \multicolumn{3}{|l|}{ Liver cancer due to NASH } \\
\hline & 1990 & 2017 & $\begin{array}{l}\text { Change } \\
(\%)\end{array}$ & 1990 & 2017 & $\begin{array}{l}\text { Change } \\
(\%)\end{array}$ \\
\hline \multicolumn{7}{|c|}{ Incidence Number } \\
\hline Both & $178,430(162,931-195,848)$ & $367,779(334,461-403,729)$ & 106.1 & $28,316(24,859-32,303)$ & $72,197(64,602-79,915)$ & 155.0 \\
\hline Female & $72,218(65,621-79,579)$ & $155,446(140,604-170,785)$ & 115.2 & $11,489(9,940-13,093)$ & $25,966(23,192-28,899)$ & 126.0 \\
\hline Male & $106,212(96,992-116,227)$ & $212,333(193,932-232,724)$ & 99.9 & $16,827(14,381-19,793)$ & $46,231(40,859-51,763)$ & 174.7 \\
\hline \multicolumn{7}{|c|}{ Prevalence Number } \\
\hline Both & $395,517,822(379,947,260-411,747,556)$ & $892,322,802(858,624,902-927,954,380)$ & 125.6 & $31,861(27,921-36,296)$ & $97,376(86,789-108,049)$ & 205.6 \\
\hline Female & $156,736,936(150,553,823-163,266,724)$ & $362,528,343(348,459,597-377,017,855)$ & 131.3 & $12,264(10,545-13,906)$ & $31,002(27,649-34,461)$ & 152.8 \\
\hline Male & $238,780,885(229,292,934-248,544,047)$ & $529,794,459(509,933,144-550,960,010)$ & 121.9 & $19,597(16,757-23,020)$ & $66,374(58,436-74,563)$ & 238.7 \\
\hline \multicolumn{7}{|c|}{ Death Number } \\
\hline Both & $61,880(55,395-67,979)$ & $118,030(108,618-128,577)$ & 90.7 & $28,528(25,037-32,528)$ & $66,875(59,560-74,511)$ & 134.4 \\
\hline Female & $26,971(23,761-29,716)$ & $49,916(45,639-55,865)$ & 85.1 & $11,963(10,389-13,638)$ & $25,741(22,883-28,696)$ & 115.2 \\
\hline Male & $34,910(30,599-38,682)$ & $68,113(61,977-74,870)$ & 95.1 & $16,566(14,132-19,493)$ & $41,134(36,391-45,989)$ & 148.3 \\
\hline \multicolumn{7}{|c|}{ DALYs Number } \\
\hline Both & $1,883,893(1,693,315-2,064,605)$ & $3,434,185(3,153,991-3,743,299)$ & 82.3 & $711,891(624,591-816,722)$ & $1,461,284(1,304,838-1,623,665)$ & 105.3 \\
\hline Female & $758,250(678,067-833,931)$ & $1,328,968(1,216,931-1,471,805)$ & 75.3 & $270,922(233,020-308,498)$ & $505,289(451,349-559,662)$ & 86.5 \\
\hline Male & $1,125,643(996,675-1,240,126)$ & $2,105,217(1,924,086-2,313,409)$ & 87.0 & $440,969(374,405-521,592)$ & $955,994(842,200-1,073,864)$ & 116.8 \\
\hline \multicolumn{7}{|c|}{ Age-standardized Incidence Rate } \\
\hline Both & $3.7(3.4-4)$ & $4.6(4.2-5.1)$ & 24.3 & $0.7(0.6-0.8)$ & $0.9(0.8-1)$ & 28.6 \\
\hline Female & $3(2.8-3.3)$ & $3.8(3.4-4.2)$ & 26.7 & $0.5(0.5-0.6)$ & $0.6(0.5-0.7)$ & 20.0 \\
\hline Male & $4.4(4.1-4.8)$ & $5.4(5-5.9)$ & 22.7 & $0.9(0.8-1)$ & $1.2(1.1-1.4)$ & 33.3 \\
\hline \multicolumn{7}{|c|}{ Age-standardized Prevalence Rate } \\
\hline Both & $8,287.2(7,972.2-8,627.8)$ & $11,060.5(10,651.3-11,493.1)$ & 33.5 & $0.8(0.7-0.9)$ & $1.2(1.1-1.3)$ & 50.0 \\
\hline Female & $6,506.1(6,250.6-6,776)$ & $8,825.7(8,481-9,179.1)$ & 35.7 & $0.6(0.5-0.6)$ & $0.7(0.6-0.8)$ & 16.7 \\
\hline Male & $10,133.7(9,751.7-10,547.2)$ & $13,352.6(12,861-13,875)$ & 31.8 & $1(0.8-1.1)$ & $1.7(1.5-1.9)$ & 70.0 \\
\hline \multicolumn{7}{|c|}{ Age-standardized Death Rate } \\
\hline Both & $1.5(1.3-1.6)$ & $1.5(1.3-1.6)$ & 0.0 & $0.7(0.6-0.8)$ & $0.8(0.8-0.9)$ & 14.3 \\
\hline Female & $1.2(1.1-1.4)$ & $1.2(1.1-1.3)$ & 0.0 & $0.6(0.5-0.6)$ & $0.6(0.5-0.7)$ & 0.0 \\
\hline Male & $1.7(1.5-1.9)$ & $1.8(1.6-1.9)$ & 5.9 & $0.9(0.8-1.1)$ & $1.1(1-1.3)$ & 22.2 \\
\hline \multicolumn{7}{|c|}{ Age-standardized DALYs Rate } \\
\hline Both & $42.4(38.1-46.5)$ & $41.8(38.5-45.5)$ & -1.4 & $16.5(14.5-18.9)$ & $18(16.1-20)$ & 9.1 \\
\hline Female & $33.5(29.9-36.8)$ & $31.6(29-35)$ & -5.7 & $12(10.4-13.7)$ & $11.9(10.6-13.2)$ & -0.8 \\
\hline Male & $51.3(45.5-56.7)$ & $52.2(47.8-57.3)$ & 1.8 & $21.3(18.2-25.2)$ & $24.6(21.8-27.6)$ & 15.5 \\
\hline
\end{tabular}

NASH: nonalcoholic steatohepatitis; DALYs: disability-adjusted life years. 


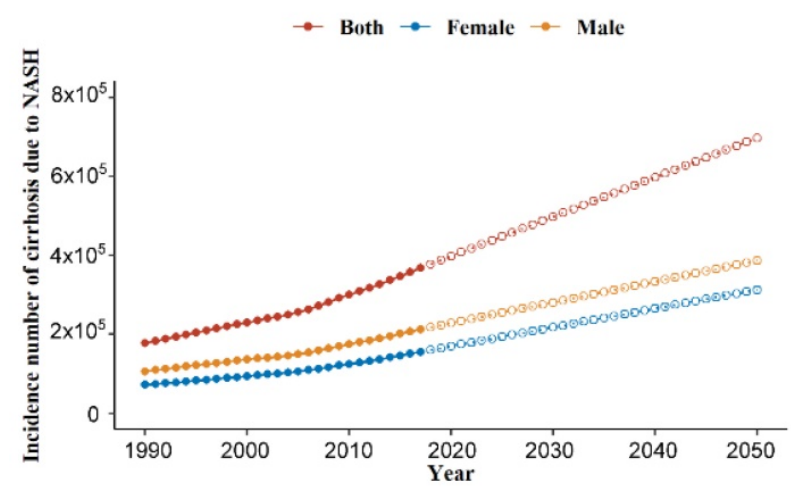

C

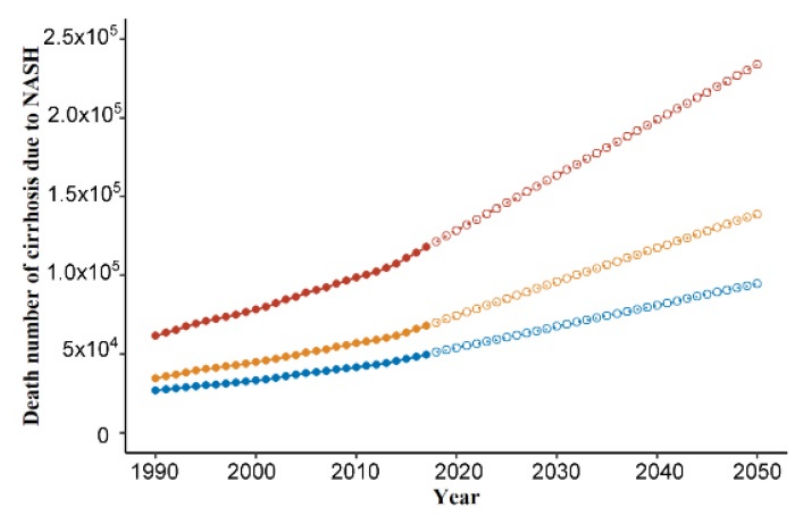

$\mathbf{E}$

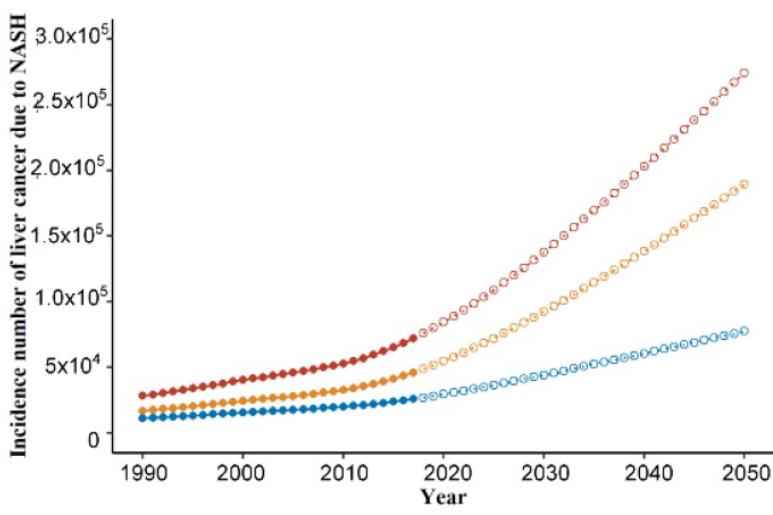

G

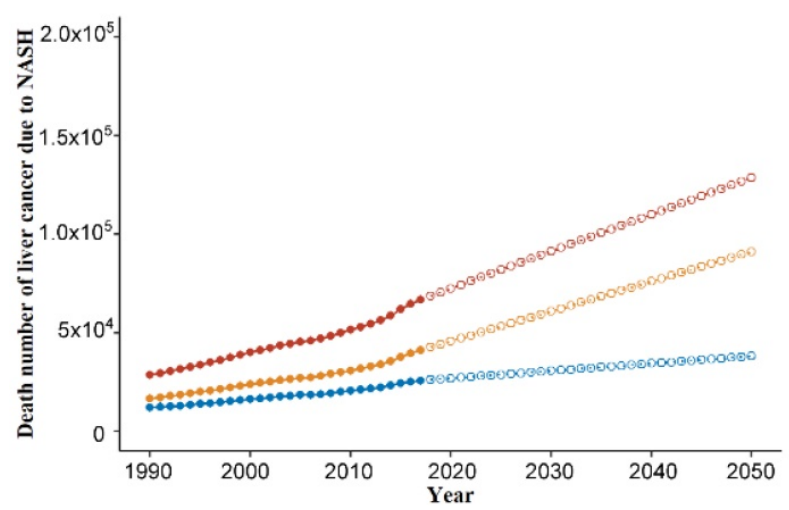

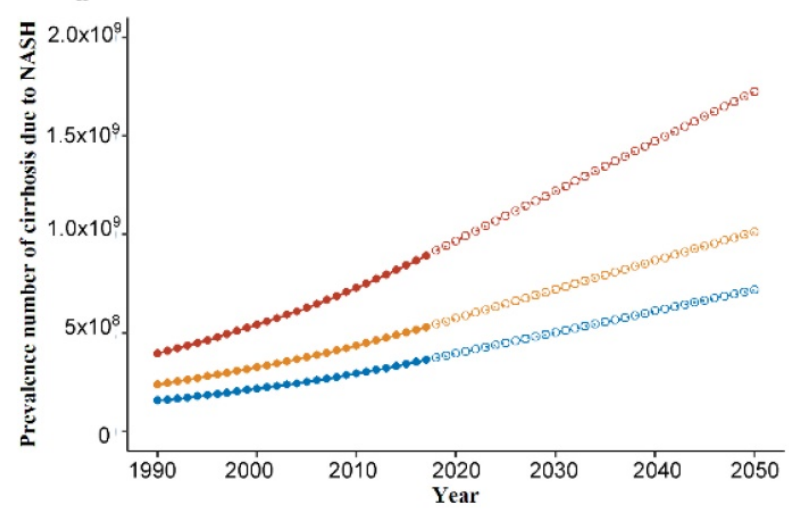

D
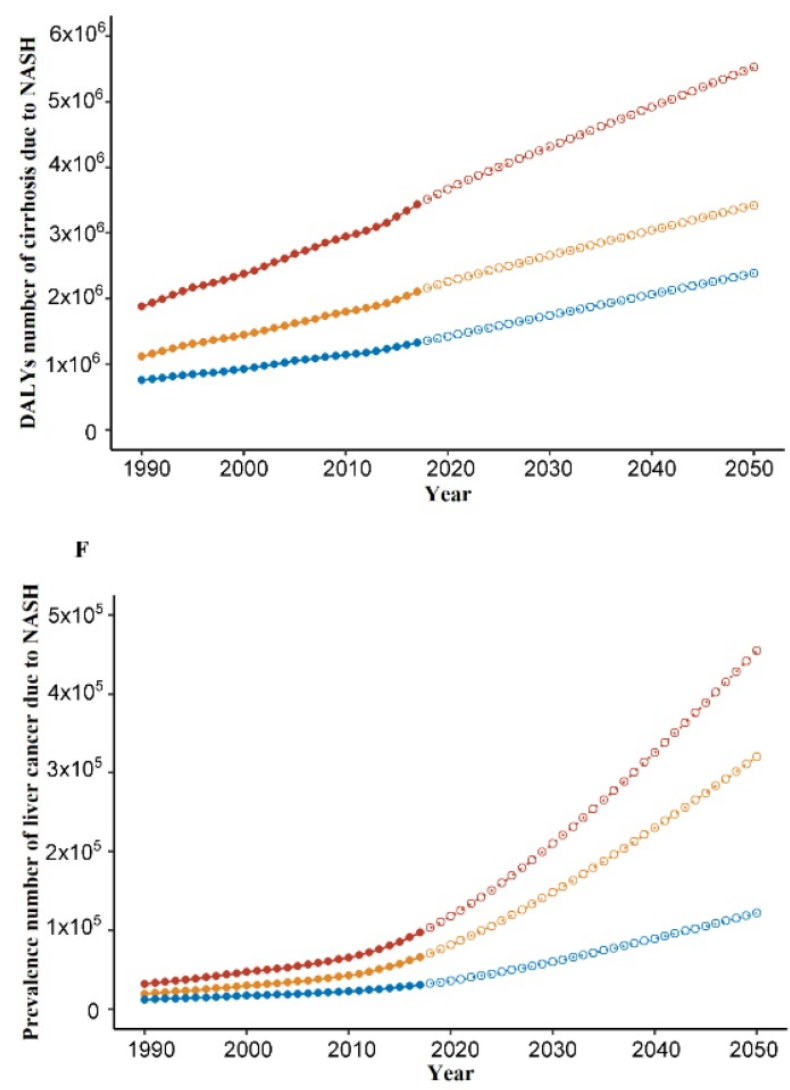

H

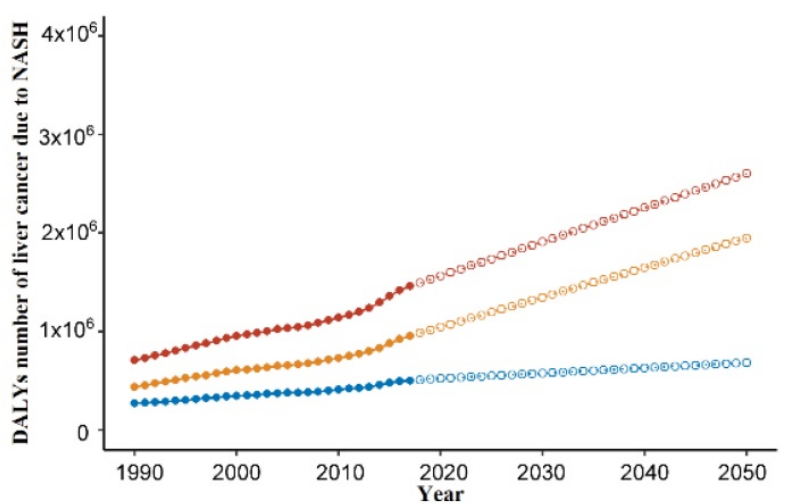

Figure 1. Global burden of cirrhosis and liver cancer due to NASH from 1990 to 2050. (A) Incidence number of cirrhosis due to NASH; (B) Prevalence number of cirrhosis due to NASH; (C) Death number of cirrhosis due to NASH; (D) DALYs number of cirrhosis due to NASH; (E) Incidence number of liver cancer due to NASH; (F) Prevalence number of liver cancer due to NASH; (G) Death number of liver cancer due to NASH; (H) DALYs number of liver cancer due to NASH. NASH: nonalcoholic steatohepatitis; DALYs: disability-adjusted life years. Dash line: forecasted NASH burden from 2018 towards 2050 through ARIMA model. 

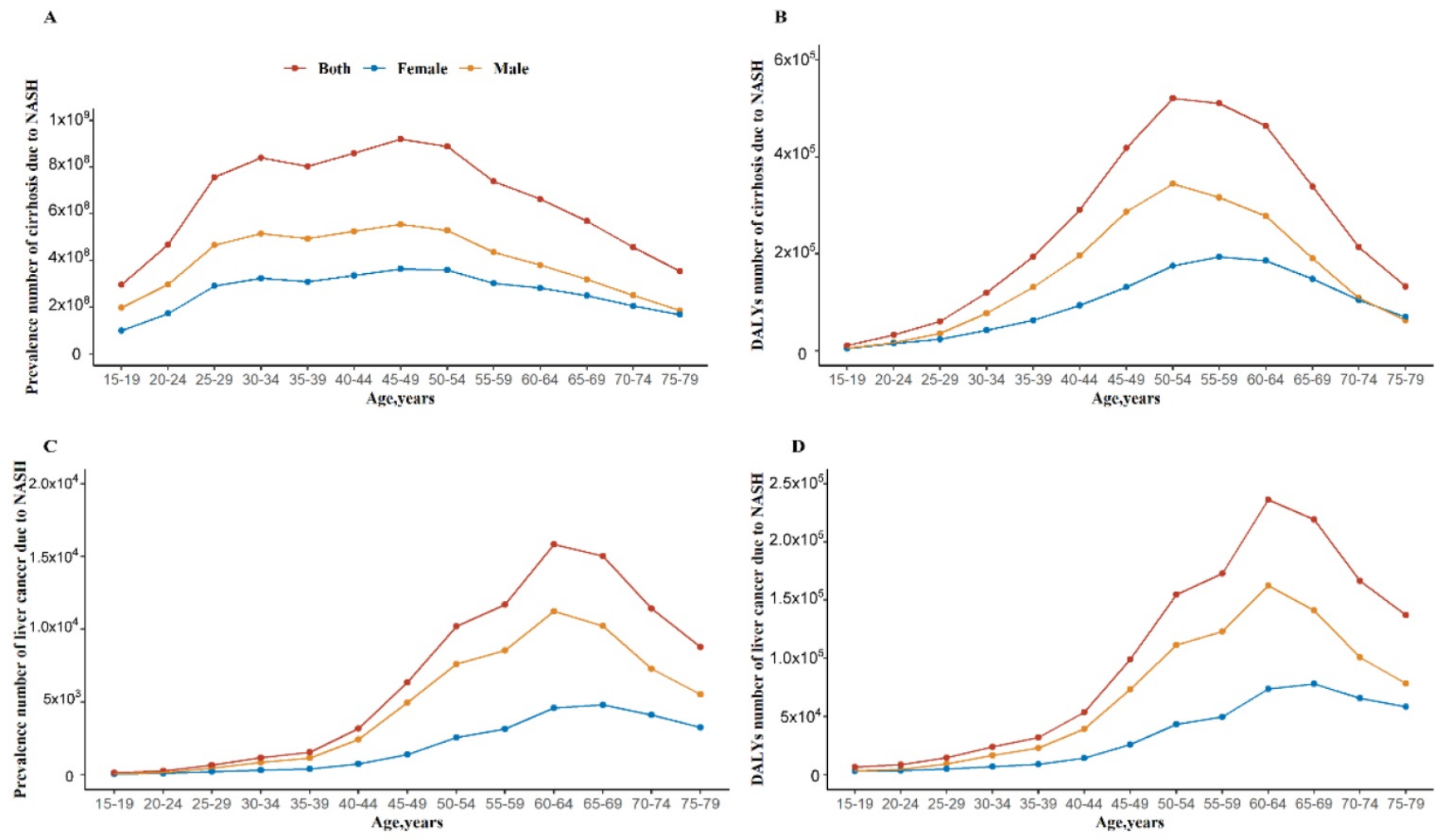

Figure 2. Global burden of cirrhosis and liver cancer due to NASH by age in 2017. (A) Prevalence number of cirrhosis due to NASH; (B) DALYs number of cirrhosis due to NASH; (C) Prevalence number of liver cancer due to NASH; (B) DALYs number of liver cancer due to NASH. NASH: nonalcoholic steatohepatitis; DALYs: disability-adjusted life years.

\section{Geographic differences in burden of cirrhosis and liver cancer due to NASH}

Figure 3A maps the distribution of health burden of cirrhosis due to NASH in 2017.The top five countries for the age-standardized DALYs rates of cirrhosis due to NASH were Egypt (239.9), Cambodia (203.2), Moldova (192.5), Guatemala (182.0), and Honduras (173.3), while the bottom five countries for the DALYs rates of cirrhosis due to NASH were Singapore (3.1), Japan (9.5), Brunei (11.3), Norway (11.9), and New Zealand (12.8).

Figure 3B maps the distribution of health burden of liver cancer due to NASH in 2017. Overall, the global distribution of health burden of cirrhosis due to NASH was different from that of liver cancer due to NASH. The top five countries for age-standardized DALYs rates of liver cancer due to NASH were Mongolia (148.2), Gambia (93.9), Mali (83.3), Guinea (81.6), and Tonga (76.5), while the bottom five countries for the DALYs rates of liver cancer due to NASH were Tunisia (3.4), Norway (3.4), Andorra (3.9), Malta (4.3), and Morocco (4.4).

\section{Burden of cirrhosis and liver cancer due to NASH by GBD regions}

We further looked at the health burden in each GBD regions in 2017 and compared with that in 1990
(Figure 4). Overall, Eastern Europe experienced the largest increase in the health burden of cirrhosis due to NASH, with the age-standardized DALYs rate increasing by $212.17 \%$, while High-income Asia Pacific experienced the largest decrease in the health burden of cirrhosis due to NASH, with the age-standardized DALY rats decreasing by $48.80 \%$.

Australasia experienced the largest increase in the health burden of liver cancer due to NASH, with the age-standardized DALYs rate increasing by $143.54 \%$, while High-income Asia Pacific experienced largest decrease in the health burden of liver cancer due to NASH, with the age-standardized DALYs rate decreasing by $39.77 \%$. Detailed data of DALYs rates of cirrhosis and liver cancer due to NASH were displayed in Table 2.

\section{Burden of cirrhosis and liver cancer due to NASH by socioeconomic status}

Bubbles in Figure 5A exhibited health burden of cirrhosis due to NASH in each country or territory with different SDI levels. The area size of each bubble represented DALYs number and $\mathrm{Y}$ coordinate indicated age-standardized DALYs rate. The highest DALYs number of cirrhosis due to NASH occurred in India $(355,010)$. Meanwhile, the highest age-standardized rates of cirrhosis due to NASH occurred in Egypt (239.9). The age-standardized rates 
of cirrhosis due to NASH showed an inverted SDI countries having the heaviest burden. U-shaped relation with SDI levels, with low-middle

\section{A}

Age-standardized DALYs rates o

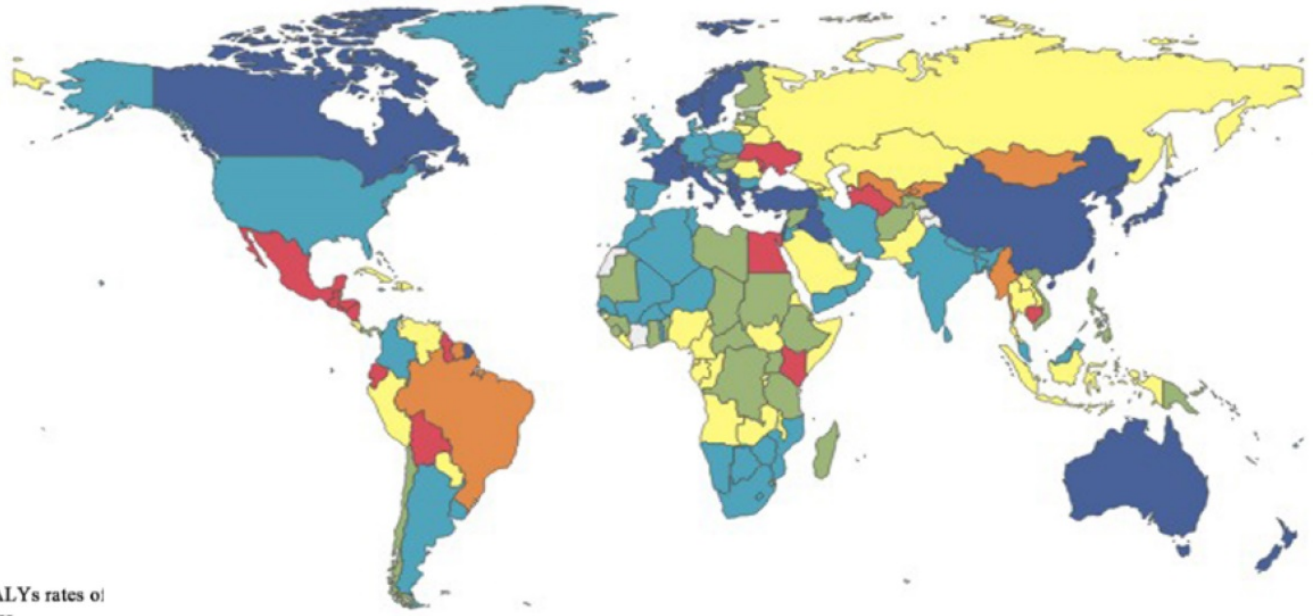
cirrhosis due to NASH
$>120$
$100 \cdot 120$
$60 \cdot 100$
$40 \cdot 60$
$25 \cdot 40$
$<25$

\section{B}



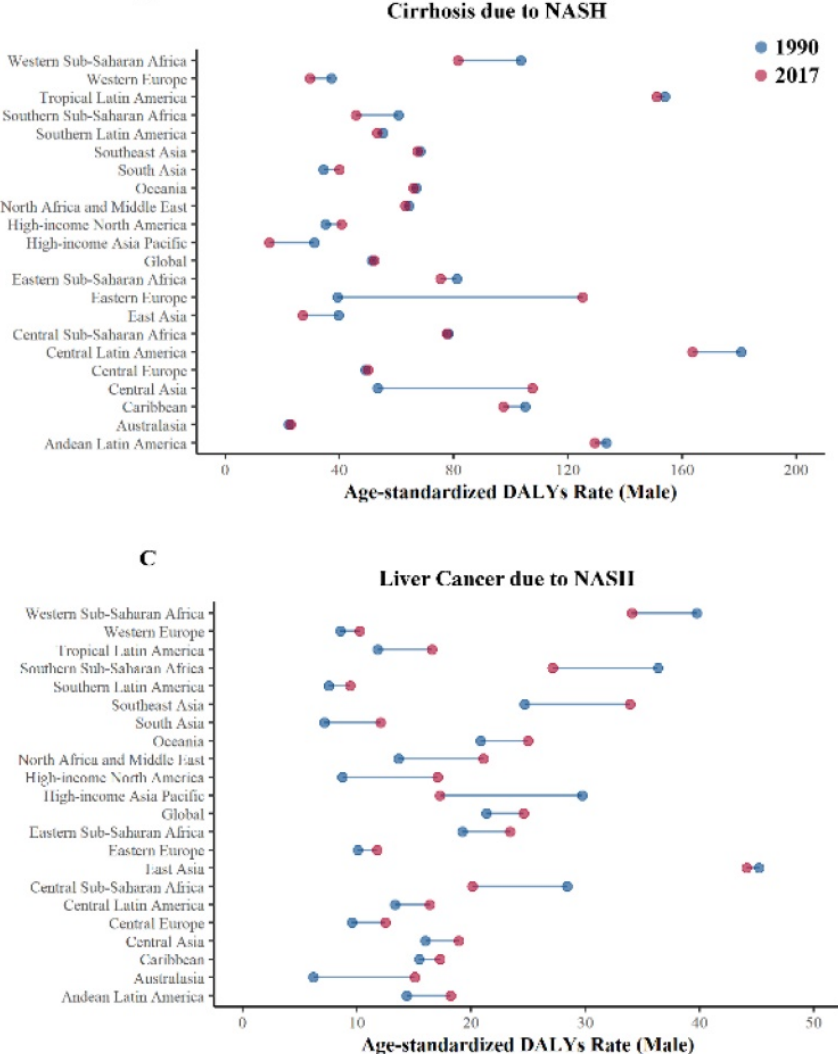

B

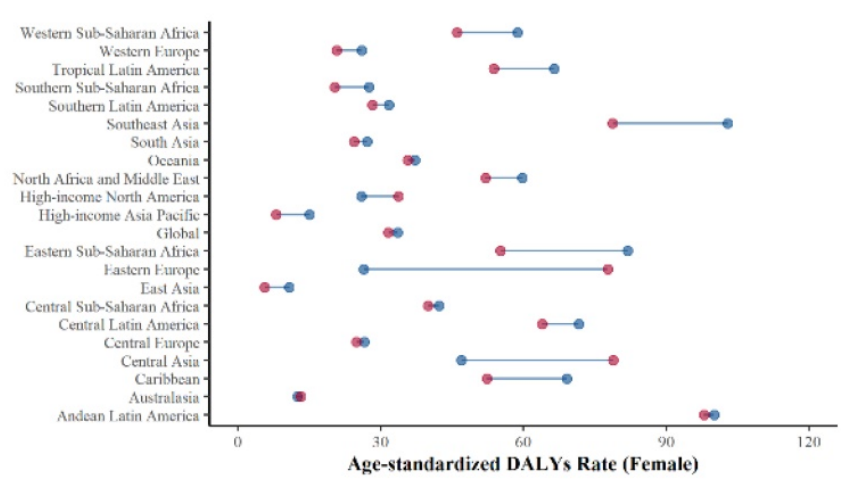

D

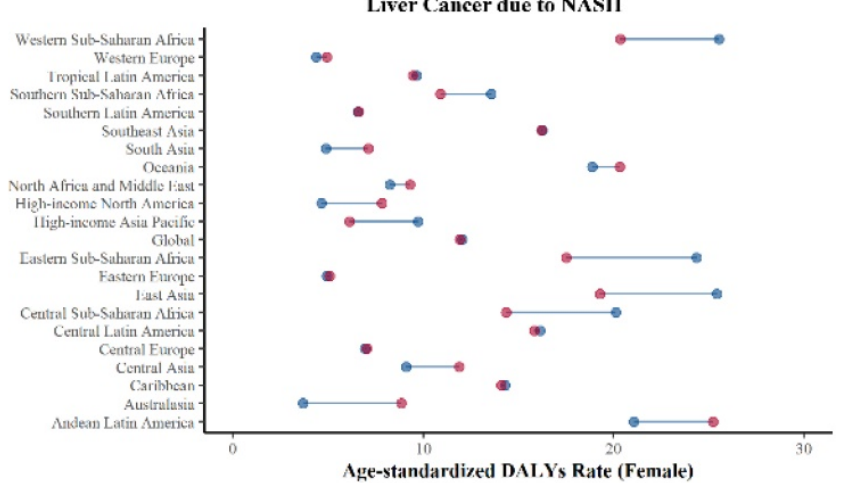

Figure 4. Health burden of cirrhosis and liver cancer due to NASH by GBD regions in 1990 and 2017. (A) Age-standardized DALYs rates of cirrhosis due to NASH (male); (B) Age-standardized DALYs rates of cirrhosis due to NASH (female); (C) Age-standardized DALYs rates of liver cancer due to NASH (male); (D) Age-standardized DALYs rates of liver cancer due to NASH (female). NASH: nonalcoholic steatohepatitis; DALYs: disability-adjusted life years.

Table 2. Age-standardized DALYs rate of NASH per 100000 persons by GBD Region in 1990 and 2017

\begin{tabular}{|c|c|c|c|c|c|c|c|c|c|c|c|c|}
\hline \multirow{3}{*}{ GBD Region } & \multicolumn{6}{|c|}{ Cirrhosis due to NASH } & \multicolumn{6}{|c|}{ Liver cancer due to NASH } \\
\hline & \multicolumn{3}{|c|}{1990} & \multicolumn{3}{|c|}{2017} & \multicolumn{3}{|c|}{1990} & \multicolumn{3}{|c|}{2017} \\
\hline & Total & Male & Female & Total & Male & Female & Total & Male & Female & Total & Male & Female \\
\hline \multicolumn{13}{|l|}{ Global } \\
\hline Global & 42.41 & 51.35 & 33.51 & 41.79 & 52.20 & 31.58 & 16.53 & 21.34 & 12.05 & 18.01 & 24.60 & 11.90 \\
\hline \multicolumn{13}{|l|}{ High income } \\
\hline High-income Asia Pacific & 22.92 & 31.16 & 15.02 & 11.73 & 15.43 & 8.01 & 18.98 & 29.74 & 9.73 & 11.43 & 17.28 & 6.12 \\
\hline Western Europe & 31.32 & 37.12 & 26.02 & 25.11 & 29.67 & 20.69 & 6.22 & 8.54 & 4.37 & 7.44 & 10.26 & 4.92 \\
\hline Australasia & 17.26 & 22.17 & 12.48 & 17.90 & 22.88 & 13.20 & 4.87 & 6.18 & 3.69 & 11.86 & 15.07 & 8.84 \\
\hline High-income North America & 30.28 & 35.05 & 25.90 & 37.08 & 40.59 & 33.74 & 6.47 & 8.72 & 4.65 & 12.21 & 17.06 & 7.81 \\
\hline Southern Latin America & 42.85 & 55.22 & 31.73 & 40.06 & 53.19 & 28.26 & 7.00 & 7.53 & 6.54 & 7.87 & 9.39 & 6.61 \\
\hline \multicolumn{13}{|c|}{ Central Europe, Eastern Europe and Central Asia } \\
\hline Central Europe & 37.15 & 49.04 & 26.46 & 36.94 & 49.86 & 24.84 & 8.11 & 9.57 & 6.93 & 9.51 & 12.49 & 7.02 \\
\hline Eastern Europe & 31.91 & 39.20 & 26.41 & 99.62 & 125.24 & 77.71 & 6.85 & 10.08 & 4.93 & 7.79 & 11.77 & 5.08 \\
\hline Central Asia & 49.92 & 53.32 & 46.89 & 92.50 & 107.62 & 78.84 & 11.97 & 16.00 & 9.08 & 14.96 & 18.91 & 11.88 \\
\hline \multicolumn{13}{|c|}{ Latin America and Caribbean } \\
\hline Tropical Latin America & 108.85 & 153.94 & 66.41 & 99.86 & 151.14 & 53.71 & 10.65 & 11.79 & 9.64 & 12.68 & 16.55 & 9.44 \\
\hline Central Latin America & 124.74 & 180.73 & 71.63 & 111.06 & 163.56 & 63.95 & 14.78 & 13.32 & 16.13 & 16.08 & 16.36 & 15.83 \\
\hline Andean Latin America & 116.54 & 133.39 & 100.12 & 113.40 & 129.39 & 97.94 & 17.81 & 14.33 & 21.07 & 21.86 & 18.21 & 25.24 \\
\hline Caribbean & 86.69 & 104.93 & 69.14 & 74.07 & 97.30 & 52.33 & 14.87 & 15.44 & 14.31 & 15.61 & 17.28 & 14.09 \\
\hline \multicolumn{13}{|c|}{ Southeast Asia, East Asia, and Oceania } \\
\hline East Asia & 25.49 & 39.57 & 10.73 & 16.31 & 27.14 & 5.54 & 35.49 & 45.21 & 25.43 & 31.60 & 44.12 & 19.29 \\
\hline Southeast Asia & 86.81 & 68.25 & 102.98 & 74.10 & 67.23 & 78.75 & 20.27 & 24.70 & 16.26 & 24.49 & 33.92 & 16.21 \\
\hline Oceania & 52.67 & 66.84 & 37.17 & 51.27 & 65.82 & 35.62 & 19.89 & 20.83 & 18.89 & 22.76 & 24.99 & 20.32 \\
\hline \multicolumn{13}{|l|}{ North Africa and Middle East } \\
\hline North Africa and Middle East & 62.11 & 64.27 & 59.72 & 57.72 & 63.04 & 51.99 & 11.00 & 13.64 & 8.23 & 15.27 & 21.09 & 9.30 \\
\hline \multicolumn{13}{|l|}{ South Asia } \\
\hline South Asia & 30.95 & 34.31 & 27.10 & 32.26 & 39.97 & 24.35 & 6.07 & 7.15 & 4.87 & 9.56 & 12.08 & 7.12 \\
\hline \multicolumn{13}{|l|}{ Sub-Saharan Africa } \\
\hline Southern Sub-Saharan Africa & 42.80 & 60.69 & 27.54 & 31.54 & 45.78 & 20.27 & 23.71 & 36.38 & 13.57 & 17.86 & 27.14 & 10.89 \\
\hline Eastern Sub-Saharan Africa & 81.47 & 81.16 & 81.90 & 65.05 & 75.27 & 55.14 & 21.82 & 19.24 & 24.37 & 20.36 & 23.40 & 17.50 \\
\hline Central Sub-Saharan Africa & 59.13 & 78.08 & 42.27 & 57.76 & 77.60 & 39.95 & 24.05 & 28.43 & 20.12 & 16.94 & 20.09 & 14.36 \\
\hline Western Sub-Saharan Africa & 82.69 & 103.44 & 58.76 & 63.06 & 81.41 & 46.00 & 32.83 & 39.77 & 25.54 & 26.97 & 34.09 & 20.35 \\
\hline
\end{tabular}

NASH: nonalcoholic steatohepatitis; DALYs: disability-adjusted life years. 


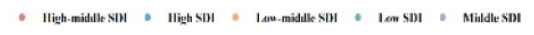

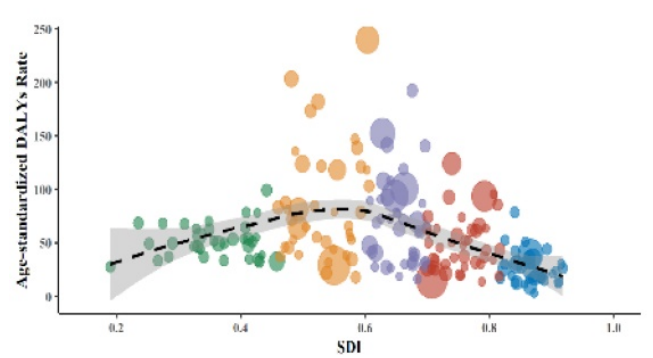

D

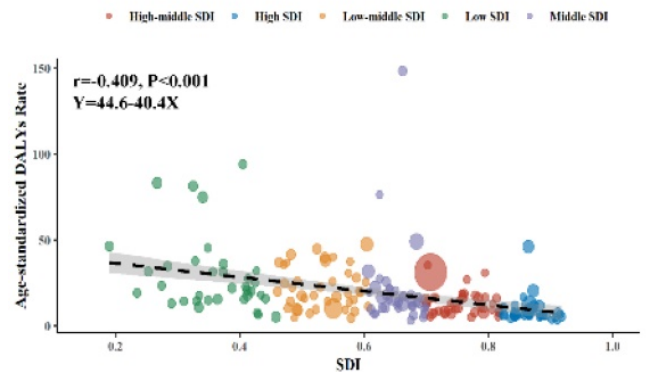

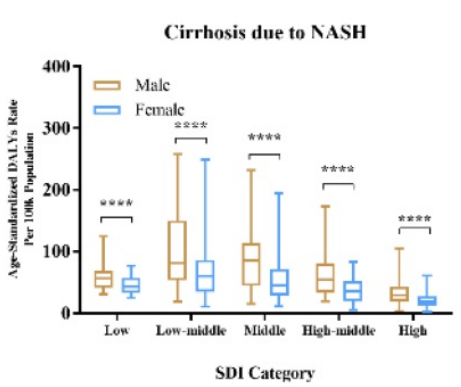

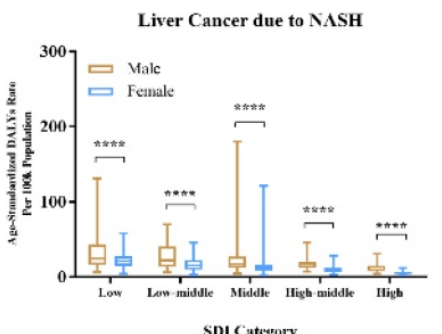

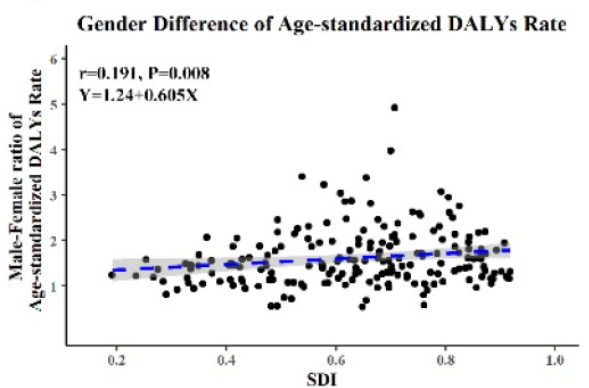

$\mathbf{F}$

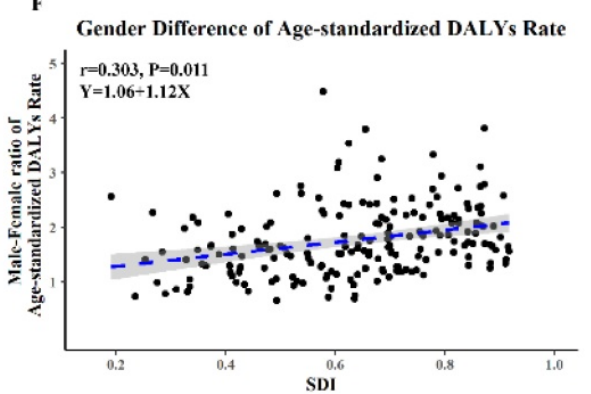

Figure 5. Global burden of cirrhosis and liver cancer due to NASH by SDI levels in 2017. (A) Age-standardized DALYs rate of cirrhosis due to NASH in 195 countries; (B) Age-standardized DALYs rate of cirrhosis due to NASH in different SDI levels; (C) Association between male-female-ratio of age-standardized DALYs rate of cirrhosis due to NASH with SDI; (D) Age-standardized DALYs rate of liver cancer due to NASH in 195 countries; (E) Age-standardized DALYs rate of liver cancer due to NASH in different SDI levels; (F) Association between male-female-ratio of age-standardized DALYs rate of liver cancer due to NASH with SDI. NASH: nonalcoholic steatohepatitis; SDI: sociodemographic index; DALYs: disability-adjusted life years. (A and D) Each circle represents a country; circles are colored according to SDI quintile; circle size corresponds to the number.

Bubbles in Figure 5D exhibited health burden of liver cancer due to NASH in each country or territory with different SDI levels. The highest DALYs number of liver cancer due to NASH occurred in China $(621,923)$. Meanwhile, the highest age-standardized rates of liver cancer due to NASH occurred in Mongolia (148.2). The age-standardized rates of liver cancer due to NASH was negatively associated with SDI levels in Pearson correlation $(\mathrm{r}=-0.409, \mathrm{p}<0.001)$ and in Linear regression (equation: $Y=44.6-40.4^{\star} X$ ).

As shown in Figure 5B, Figure 5E, male had higher age-standardized DALYs rates of cirrhosis and liver cancer due to NASH across all SDI categories. Differences by sex in age-standardized DALYs rates of cirrhosis due to NASH were positively associated with SDI in Pearson correlation $(\mathrm{r}=0.191, \mathrm{p}=0.008)$ and in Linear regression (equation: $Y=1.24+0.605^{*} \mathrm{X}$ ) (Figure 5C). Besides, differences by sex in age-standardized DALYs rates of liver cancer due to NASH were positively associated with SDI as well in Pearson correlation $(\mathrm{r}=0.303, \mathrm{p}=0.011)$ and in Linear regression (equation: $\mathrm{Y}=1.06+1.12^{*} \mathrm{X}$ ) (Figure 5F).

\section{Discussion}

The present study provides an overview of the global pattern of the health burden of cirrhosis and liver cancer due to NASH by year, gender, age, region, and socioeconomic levels. We found that the incidence number, prevalence number, death number,
DALYs number, age-standardized incidence rate and age-standardized prevalence rate were increasing over time. The age-standardized rates of cirrhosis due to NASH showed an inverted U-shaped relation with SDI levels, with low-middle SDI countries having the heaviest burden. The age-standardized rates of liver cancer due to NASH was negatively associated with SDI levels. Gender disparity has existed since 1990, with male being more heavily impacted. Malefemale-ratio of age-standardized DALYs rates in liver cancer due to NASH were positively related to SDI.

From 1999 to 2017, the global age-standardized incidence and prevalence rates of cirrhosis and liver cancer due to NASH increased dramatically, and measurements would keep rising if without further effective interventions, paralleling the dramatic modifications in lifestyle and the worldwide increase in obesity [18]. Recent age-adjusted estimates of global obesity prevalence reported that at least $30 \%$ of male and $35 \%$ of female are obese in many countries worldwide [19]. Conversely, the global agestandardized death and DALYs rates of cirrhosis and liver cancer due to NASH were not increasing at the same rate, which might be associated with recent advances in early diagnosis and therapy of cirrhosis and liver cancer [20, 21]. It is worth noting, however, that there was an upward trend in the global age-standardized death and DALYs rates of liver cancer due to NASH in males according to ARIMA 
projection model. Despite all that, the global absolute numbers of incidence, prevalence, death and DALYs increased continuously from 1990 to 2017, which was probably related to population growth and aging [22].

Our study observed socioeconomic disparity in the burden of cirrhosis and liver cancer due to NASH. The age-standardized rates of cirrhosis due to NASH showed an inverted U-shaped relation with SDI levels, with low-middle SDI countries having the heaviest burden. The age-standardized rates of liver cancer due to NASH was negatively associated with SDI levels. One possible explanation might be that low SDI countries and low-middle SDI countries are undergoing a period of rapid economic and social changes, with trends towards urbanization and dramatic changes in diets and lifestyles, and these factors might increase the risks of obesity, NAFLD, and NASH, while people in high-SDI countries are more likely to have healthy diet habits [23, 24]. What's more, with rapid urbanization in developing countries, air pollution caused by multiple-pollutant emissions and vehicle exhaust has been aggravated year by year. Compared with developed countries who were experienced in dealing with air pollution, developing countries may be less experienced. An increasing number of studies have shown that air pollution containing particulate matter $(\mathrm{PM}) \leq 2.5 \mu \mathrm{m}$ (PM2.5) plays an important role in the development of NAFLD [25]. Evidence from a Taiwanese perspective cohort Study (REVEAL-HBV) further showed that long-term PM2.5 exposure increased the risk for liver cancer [26].

It should be noted that the global distribution of health burden of cirrhosis due to NASH was different from that of liver cancer due to NASH. For example, although the age-standardized DALYs rate of cirrhosis due to NASH in China ranked 185th from high to low, the age-standardized DALYs rate of liver cancer due to NASH in China ranked 30th around the world. Thus, there might be underlying social and medical explanation. NAFLD-related liver cancer may also occur in non-cirrhotic livers. In a study from the Veterans Administration system, $13 \%$ of patients with liver cancer did not have cirrhosis, which was different from the traditional NAFLD-NASHcirrhosis-hepatocellular carcinoma sequence [27, 28]. Our findings suggested that liver cancer in NASH without cirrhosis should be paid attention to in future. Besides, coexisting conditions such as Hepatitis B Virus (HBV) infection and aflatoxin intake in China may accelerate the phenomenon [29, 30]. Another possible explanation was that cirrhosis due to NASH may be underestimated caused by poor patient acceptability of liver biopsy during compensation and inaccuracy of noninvasive methods to diagnose
NASH and cirrhosis [31]. With an increasing health burden of NASH-related end-stage liver disease, there is an urgent need to develop accurate noninvasive methods for diagnosis of NAFLD and NASH and monitoring of fibrosis progression.

We observed gender disparity in cirrhosis and liver cancer burden due to NASH by year, age, and different SDI levels. Extensive evidence suggested that the prevalence of NAFLD/NASH in male was higher than that in female, especially female of fertile age [32]. A large cross-sectional population-based survey in 39,151 Japanese subjects (health check-up) with the mean age of 45.8 years showed that the prevalence of NAFLD was $26 \%$ in Male and $12.7 \%$ in female [33]. After menopause female display a similar or even a higher DALYs number of cirrhosis due to NASH compared to male after 70 years old, a finding supporting a protective effect of estrogens [34]. Data in rats and human models suggested that high estrogen level is negatively associated with hepatic steatosis through changes in gene expression of molecules related to fat oxidation and lipogenesis [32]. What's more, although the global prevalence of obesity in female $(6 \%-15 \%)$ was higher than that in male (3\%-11\%), male had more visceral fat than female, which also explained the heavier disease burden of male than that of female [32, 35]. Understanding the epidemiology of NAFLD/NASH is helpful to interpret gender disparity in the health burden of cirrhosis and liver cancer burden due to NASH. On the other hand, the role of gender in fibrosis progression in NAFLD/NASH is still multifaceted, although some studies have reported that the risk was significantly increased in male than in female [36]. In addition, the burden of liver cancer due to NASH was higher in male than female also probably be due to the tumorigenic effect of androgens $[37,38]$.

The strengths of our study were the comprehensive estimations of burden of cirrhosis and liver cancer as measured with incidence, prevalence, death, and DALYs from 1990 to 2017, and the projection for 2018 towards 2050. It is worth-mentioning that DALYs, which were useful for quantifying and ranking disease burden, were thoroughly studied in our research. DALYs were generated by summing years of life lost due to premature death and years lived with disability. The years of life lost represent the years lost due to premature death; the years live with disability represents the years live with cirrhosis and liver cancer due to NASH. DALYs reflects the actual health status [10]. As shown earlier, in our research, the global age-standardized prevalence rates of cirrhosis and liver cancer due to NASH increased from 1990 to 
2017, while the global age-standardized DALYs rates of cirrhosis and liver cancer due to NASH were not increasing, which may be due to recent advances in early diagnosis and therapy of cirrhosis and liver cancer [20, 21] and could help decision makers understand the importance of paying attention to cirrhosis and liver cancer due to NASH.

However, there were several limitations of this study. First, our analysis shared the limitations of GBD estimates [11-16]. The accuracy of the GBD estimates was limited by the quality and availability of original hospital and claims data, and the method of data processing may inevitably introduce bias. Second, cases due to NASH were defined only if $\mathrm{NASH}$ was listed as a specific etiology in the manuscript. Liver biopsy is still gold standard of NASH and fibrosis progression, with limitations including invasiveness, poor acceptability and sampling variability, which may cause underestimation of NASH and cirrhosis. Third, patients admitted to hospitals mostly had decompensated cirrhosis, thus number of compensated cirrhosis may be underestimated. Fourth, subgroup analyses by with or without alcoholic hepatitis and virus hepatitis were not performed. Notwithstanding the above limitations, the findings of this study could serve as an impetus for continued efforts toward eliminating cirrhosis and liver cancer due to NASH.

\section{Conclusion}

In conclusion, the global, comparative study demonstrated the trend of health burden of cirrhosis and liver cancer due to NASH by age, gender, location and socioeconomic levels. Cirrhosis and liver cancer due to NASH was becoming a serious global public health problem and would keep worsening if without interventions. Male had a higher health burden compared with that of female. Geographic influences existed, while low SDI countries and low-middle SDI countries tended to bear more burden. These findings will hopefully raise awareness of cirrhosis and liver cancer due to NASH and reveal an urgent need for prevention of NASH.

\section{Abbreviations}

NASH: Nonalcoholic Steatohepatitis; GBD: Global Burden of Disease; SDI: Socio-demographic Index; DALYs: disability-adjusted life years; NAFLD: Nonalcoholic fatty liver disease; TFU25: total fertility rate under the age of 25; EDU15+: education for those aged 15 and older; LDI: lag distributed income; ARIMA: Auto-Regressive Integrated Moving Average; UI: uncertainty interval; PM: particulate matter; HBV: Hepatitis B Virus.

\section{Supplementary Material}

Supplementary figures and tables.

http://www.jcancer.org/v12p2855s1.pdf

\section{Acknowledgements}

The estimates used in this manuscript based on the GBD data and methodologies. We appreciate the visionary global health leadership of the Institute for Health Metrics and Evaluation (IHME) in Seattle, Washington, and the contribution of all anonymous collaborators, without whom this report would not be possible.

\section{Funding}

This work was supported in part by grants from the National Natural Science Foundation of China (81870564, 81670744), and Science Technology Department of Zhejiang Province of China (No. 2017C33037). The authors declare that there is no other potential conflict of interests.

\section{Ethics Committee Approval and Patient Consent}

Ethical approval and informed consent were not required for this study because of public accessibility of the data.

\section{Competing Interests}

The authors have declared that no competing interest exists.

\section{References}

1. Suzuki A, Diehl AM. Nonalcoholic Steatohepatitis. Annu Rev Med. 2017; 68: 85-98.

2. Chooi YC, Ding C, Magkos F. The epidemiology of obesity. Metabolism. 2019; 92: 6-10.

3. Younossi ZM, Koenig AB, Abdelatif D, et al. Global epidemiology of nonalcoholic fatty liver disease-Meta-analytic assessment of prevalence, incidence, and outcomes. Hepatology. 2016; 64: 73-84.

4. Chalasani N, Younossi Z, Lavine JE, et al. The diagnosis and management of nonalcoholic fatty liver disease: Practice guidance from the American Association for the Study of Liver Diseases. Hepatology. 2018; 67: 328-57.

5. Sepanlou SG, Safiri S, Bisignano C, et al. The global, regional, and national burden of cirrhosis by cause in 195 countries and territories, 1990-2017: a systematic analysis for the Global Burden of Disease Study 2017. The Lancet Gastroenterology \& Hepatology. 2020; 5: 245-66.

6. Paik JM, Golabi P, Younossi Y, et al. Changes in the Global Burden of Chronic Liver Diseases From 2012 to 2017: The Growing Impact of NAFLD. Hepatology. 2020; 72: 1605-16.

7. Sanyal AJ, Harrison SA, Ratziu V, et al. The Natural History of Advanced Fibrosis Due to Nonalcoholic Steatohepatitis: Data From the Simtuzumab Trials. Hepatology. 2019; 70: 1913-27.

8. Chen J, Talwalkar JA, Yin M, et al. Early detection of nonalcoholic steatohepatitis in patients with nonalcoholic fatty liver disease by using MR elastography. Radiology. 2011; 259: 749-56.

9. Perumpail BJ, Khan MA, Yoo ER, et al. Clinical epidemiology and disease burden of nonalcoholic fatty liver disease. World J Gastroenterol. 2017; 23: 8263-76.

10. GBD. Global, regional, and national disability-adjusted life-years (DALYs) for 359 diseases and injuries and healthy life expectancy (HALE) for 195 countries and territories, 1990-2017: a systematic analysis for the Global Burden of Disease Study 2017. Lancet. 2018; 392: 1859-922.

11. James SL, Abate D, Abate KH, et al. Global, regional, and national incidence, prevalence, and years lived with disability for 354 diseases and injuries for 195 countries and territories, 1990-2017: a systematic analysis for the Global Burden of Disease Study 2017. The Lancet. 2018; 392: 1789-858. 
12. Murray CJL, Callender CSKH, Kulikoff XR, et al. Population and fertility by age and sex for 195 countries and territories, 1950-2017: a systematic analysis for the Global Burden of Disease Study 2017. The Lancet. 2018; 392: 1995-2051.

13. Dicker D, Nguyen G, Abate D, et al. Global, regional, and national age-sexspecific mortality and life expectancy, 1950-2017: a systematic analysis for the Global Burden of Disease Study 2017. The Lancet. 2018; 392: 1684-735.

14. Stanaway JD, Afshin A, Gakidou E, et al. Global, regional, and national comparative risk assessment of 84 behavioural, environmental and occupational, and metabolic risks or clusters of risks for 195 countries and territories, 1990-2017: a systematic analysis for the Global Burden of Disease Study 2017. The Lancet. 2018; 392: 1923-94.

15. Lozano R, Fullman N, Abate D, et al. Measuring progress from 1990 to 2017 and projecting attainment to 2030 of the health-related Sustainable Development Goals for 195 countries and territories: a systematic analysis for the Global Burden of Disease Study 2017. The Lancet. 2018; 392: 2091-138.

16. GBD. Global, regional, and national age-sex-specific mortality for 282 causes of death in 195 countries and territories, 1980-2017: a systematic analysis for the Global Burden of Disease Study 2017. Lancet. 2018; 392: 1736-88.

17. Foreman KJ, Marquez N, Dolgert A, et al. Forecasting life expectancy, years of life lost, and all-cause and cause-specific mortality for 250 causes of death: reference and alternative scenarios for 2016-40 for 195 countries and territories. Lancet. 2018; 392: 2052-90.

18. Lonardo A, Byrne CD, Caldwell SH, et al. Global epidemiology of nonalcoholic fatty liver disease: Meta-analytic assessment of prevalence, incidence, and outcomes. Hepatology. 2016; 64: 1388-9.

19. Afshin A, Forouzanfar MH, Reitsma MB, et al. Health Effects of Overweight and Obesity in 195 Countries over 25 Years. N Engl J Med. 2017; 377: 13-27.

20. Ge PS, Runyon BA. Treatment of Patients with Cirrhosis. N Engl J Med. 2016; 375: 2104-5.

21. Forner A, Reig M, Bruix J. Hepatocellular carcinoma. The Lancet. 2018; 391: 1301-14

22. [Iternet] United Nations. Department of Economic and Social Affairs. Population Division. World Population Prospects, the 2017 Revision. Available at: http://esa.un.org/unpd/wpp/. Accessed January 1, 2019.

23. Estes C, Anstee QM, Arias-Loste MT, et al. Modeling NAFLD disease burden in China, France, Germany, Italy, Japan, Spain, United Kingdom, and United States for the period 2016-2030. J Hepatol. 2018; 69: 896-904.

24. Mahady SE, George J. Predicting the future burden of NAFLD and NASH. J Hepatol. 2018; 69: 774-5.

25. Xu MX, Ge CX, Qin YT, et al. Prolonged PM2.5 exposure elevates risk of oxidative stress-driven nonalcoholic fatty liver disease by triggering increase of dyslipidemia. Free Radic Biol Med. 2019; 130: 542-56.

26. Pan $\mathrm{WC}, \mathrm{Wu} \mathrm{CD}, \mathrm{Chen} \mathrm{MJ}$, et al. Fine Particle Pollution, Alanine Transaminase, and Liver Cancer: A Taiwanese Prospective Cohort Study (REVEAL-HBV). Jnci-J Natl Cancer I. 2016; 108

27. Younossi Z, Henry L. Contribution of Alcoholic and Nonalcoholic Fatty Liver Disease to the Burden of Liver-Related Morbidity and Mortality. Gastroenterology. 2016; 150: 1778-85.

28. Welzel TM, Graubard BI, Zeuzem S, et al. Metabolic syndrome increases the risk of primary liver cancer in the United States: a study in the SEER-Medicare database. Hepatology. 2011; 54: 463-71.

29. Wang FS, Fan JG, Zhang Z, et al. The Global Burden of Liver Disease: The Major Impact of China. Hepatology. 2014; 60: 2099-108

30. Chen JG, Zhang SW. Liver cancer epidemic in China: Past, present and future. Semin Cancer Biol. 2011; 21: 59-69.

31. Castera L, Friedrich-Rust M, Loomba R. Noninvasive Assessment of Liver Disease in Patients With Nonalcoholic Fatty Liver Disease. Gastroenterology. 2019; 156: 1264-81.e4.

32. Ballestri S, Nascimbeni F, Baldelli E, et al. NAFLD as a Sexual Dimorphic Disease: Role of Gender and Reproductive Status in the Development and Progression of Nonalcoholic Fatty Liver Disease and Inherent Cardiovascular Risk. Adv Ther. 2017; 34: 1291-326.

33. Kojima S, Watanabe N, Numata M, et al. Increase in the prevalence of fatty liver in Japan over the past 12 years: analysis of clinical background. J Gastroenterol. 2003; 38: 954-61.

34. $\mathrm{Xi} \mathrm{B}$, Liang $\mathrm{Y}, \mathrm{He} \mathrm{T}$, et al. Secular trends in the prevalence of general and abdominal obesity among Chinese adults, 1993-2009. Obes Rev. 2012; 13: 287-96.

35. Jaacks LM, Vandevijvere S, Pan A, et al. The obesity transition: stages of the global epidemic. Lancet Diabetes Endocrinol. 2019; 7: 231-40.

36. Bedogni G, Miglioli L, Masutti F, et al. Incidence and natural course of fatty liver in the general population: the Dionysos study. Hepatology. 2007; 46: 1387-91.

37. Fattovich G, Stroffolini T, Zagni I, et al. Hepatocellular carcinoma in cirrhosis: incidence and risk factors. Gastroenterology. 2004; 127: S35-50.

38. Zhang H, Li XX, Yang $Y$, et al. Significance and mechanism of androgen receptor overexpression and androgen receptor/mechanistic target of rapamycin cross-talk in hepatocellular carcinoma. Hepatology. 2018; 67: 2271-86 